\title{
Rapid identification of an A1555G mutation in human mitochondrial DNA implicated in aminoglycoside-induced ototoxicity
}

Received: March 5, 1999 / Accepted: June 4, 1999

\begin{abstract}
This article describes a multiplex allele-specific PCR (AS-PCR) approach for detection of an A to G mutation occurring in the human mitochondrial 12s RNA gene at nucleotide 1555. Possession of this mutation has been shown to be associated with irreversible hearing loss following administration of aminoglycoside antibiotics, and in some families is associated with profound sensorineural deafness in the absence of aminoglycoside antibiotics. We screened 206 unrelated individuals from the province of Otago, New Zealand, and found one who possessed the mitochondrial 1555 A to $\mathrm{G}$ mutation $(0.48 \%$; $95 \%$ confidence interval, 0.01-2.75).
\end{abstract}

Key words Aminoglycoside-induced ototoxicity - Mitochondrial DNA · Multiplex allele-specific polymerase chain reaction

\section{Introduction}

Administration of aminoglycoside antibiotics is associated with the dual risk of hearing loss and renal damage. While high doses of aminoglycosides virtually ensure hearing loss, not everyone is at equal risk of aminoglycoside-induced hearing loss at lower drug doses. Since the suggestion that some individuals appear more susceptible to aminoglycoside-induced hearing loss than others and the demonstration of a maternal mode of transmission of this susceptibility, a single base mutation associated with

B.J. Scrimshaw $\cdot$ J.M. Faed $\cdot$ K. Yun $(\bowtie)$

Department of Pathology, Dunedin School of Medicine, University of Otago, P.O. Box 913, Dunedin, New Zealand

Tel. +64-3-479-7172; Fax +64-3-479-7136

e-mail: han-seung.yoon@stonebow.otago.ac.nz

W.P. Tate

Department of Biochemistry, University of Otago, Dunedin, New Zealand aminoglycoside-induced deafness has been identified (Higashi 1989; Prezant et al. 1993). The identified mutation is a single-base substitution, adenosine to guanosine, that occurs in the mitochondrial 12s ribosomal RNA gene at position 1555 (A1555G mutation). In addition to its association with aminoglycoside-induced deafness, this A1555G mutation is also implicated in maternally inherited hearing loss in the absence of aminoglycoside exposure in some families (Prezant et al. 1993; Marthijs et al. 1996; ElSchahawi et al. 1997).

Internationally, population studies to ascertain the frequency of the A1555G mutation have revealed it to be relatively rare. The mutation was absent in each of 100 American (Usami et al. 1997), 400 Mongolian (Pandya et al. 1997), and 414 ethnically diverse subjects (Hutchin et al. 1993). Of patients with aminoglycoside-induced deafness, all patients from 12 Spanish families who had received aminoglycosides (Estivill et al. 1998), 28 of 32 subjects from 5 Japanese families with a history of aminoglycoside-induced hearing loss (Usami et al. 1997), yet only 7 of 41 American patients with aminoglycoside-induced hearing loss (Fischel-Ghodsian et al. 1997) were found to possess the mutation. These findings have raised the suggestion that the mutation is more frequently encountered in Asian populations.

Effective prevention of aminoglycoside-induced hearing loss requires screening of large population groups to identify subjects at risk and will only be feasible if reliable and cost-effective methods are available. Here we report a rapid and simple method for large-scale screening of the A1555G mutation by allele-specific PCR (AS-PCR).

\section{Methods and materials}

This approach uses four oligonucleotide primers that result in two amplification products (Fig. 1). One pair of oligonucleotide primers flanks the A1555G mutation site and allows amplification of a common fragment regardless of the presence or absence of the mutation. Two allele-specific oligonucleotide primers, each having a mismatch in their 3'- 
Fig. 1. Schematic diagram representing the multiplex allelespecific PCR design. A common amplification product (519bp) results from amplification with primers Otox-F and 16s- $\mathrm{R}$ regardless of the presence of the mutation; primer Mut-R anneals only to the $1555 \mathrm{~A}$ to $\mathrm{G}$ mutation allele and allows amplification of a 324bp amplification product with primer Otox-F. In the absence of this mutation, primer Norm-F allows amplification of a 241-bp product with primer $16 \mathrm{~s}-\mathrm{R}$
Otox-F $16 s-R$

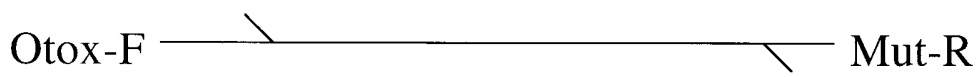

Mutant allele amplification product $(324 \mathrm{bp})$

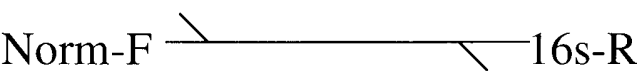

Normal amplification product (241bp)

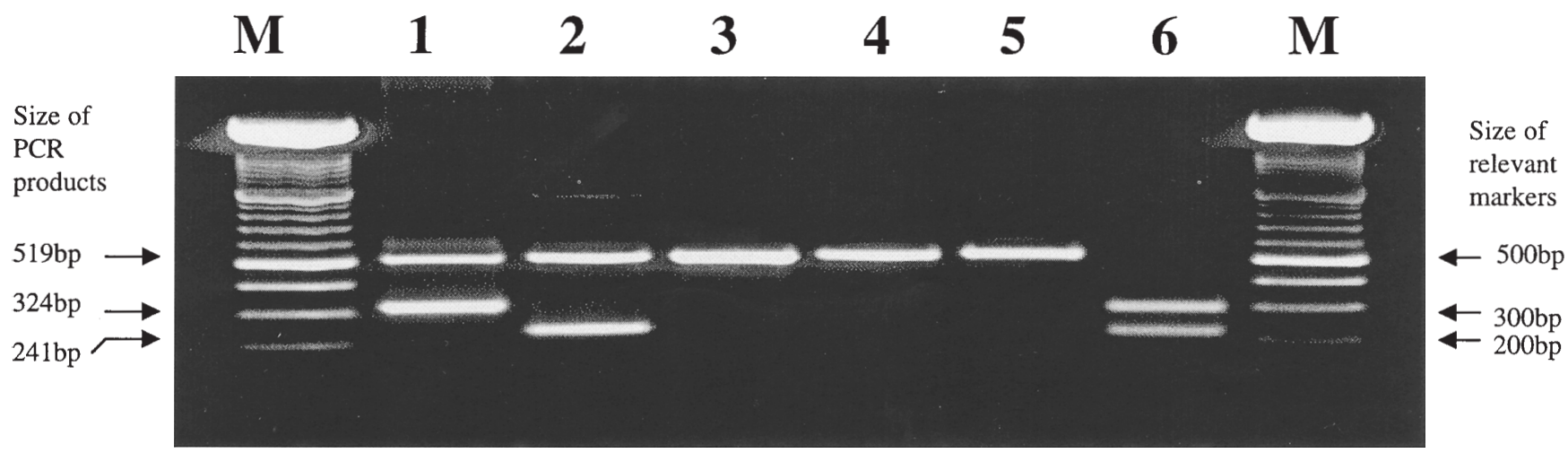

Fig. 2. Agarose gel electrophoresis. Multiplex AS-PCR of samples in which the $1555 \mathrm{~A}$ to $\mathrm{G}$ mutation is present (lane 1) or absent (lane 2). Lanes 3 and 4 show amplification of the common 519-bp fragment from the same samples using only primers Otox-F and 16s-R. Alw26I cleav- age of the samples in lanes 3 and 4 is shown in lanes 5 and 6 . The sample in lane 5 possesses the mutation that deletes a cleavage site for the restriction enzyme Alw26I. Lane M contains a 100-bp ladder molecular weight marker most base, are designed to anneal to both the forward and reverse sequence. These primers allow amplification either of a fragment that contains the mutation or a fragment that does not. The difference in size of these amplification products can be detected on an agarose gel and thus allows rapid identification of the presence or absence of the mutation.

Briefly, primers Otox-F (TATATACCGCCATCTTCAGC) and Mut-R (CACTTACCATGTTACGACTTGC) allow amplification of a 324-bp product from only mutant human mitochondrial DNA because primer Mut-R possesses a mismatch in its $3^{\prime}$-most base that is specific only for the mutant allele (Fig. 1). Likewise, primers 16S-R (GCGCCAGGTTTCAATTTCTATCG) and Norm-F (CCTACGCATTTATATAGAGGAGA) allow amplification of a 241-bp product from only the normal sequence because of a $3^{\prime}$-mismatch in primer Norm-F that is specific for only the normal allele. Primers Otox-F and 16s-R allow amplification of a 519-bp product from both normal and mutant human mitochondrial DNA. PCR reaction mixtures had a final concentration of 50ng genomic DNA, $0.2 \mathrm{mM}$ of each dNTP, $250 \mathrm{nM}$ of Mut-R, 250nM of Norm-F, 1,000nM of Otox-F, 1,000nM of 16s-R, 0.5 U Amplitaq Gold (Perkin-
Elmer, Norwalk, CT, USA) in $1 \times$ the manufacturer's PCR reaction buffer. Amplification consisted of 35 cycles of $94^{\circ} \mathrm{C}$ denaturation, $60^{\circ} \mathrm{C}$ annealing, and $72^{\circ} \mathrm{C}$ extension, each step being of 30 s duration.

To accretion the methodology, we first applied the ASPCR to a 318 cell line (EBV-transformed lymphoblastic cells), kindly provided by Dr. N. Fischel-Ghodsian, which was derived from a patient with the A1555G mutation (Prezant et al. 1993). AS-PCR products were then subjected to direct sequencing to confirm the genotype (data not shown). After establishment of the AS-PCR method, we screened 206 unrelated individuals from the province of Otago, New Zealand.

\section{Results and discussion}

Multiplex AS-PCR from individuals who possess the A1555G mutation results in PCR products that are 519bp and $324 \mathrm{bp}$ in length (Fig. 2; lane 1), while amplification from individuals without the mutation results in PCR prod- 
ucts that are 519 and 241 bp in length (Fig. 2; lane 2). The A1555G mutation detected by this assay deletes a restriction cleavage site for the enzyme Alw26I. In addition to direct sequencing, to confirm the reliability of this multiplex AS-PCR assay, we amplified the 519-bp common amplification fragment from the same DNA samples using only primers Otox-F and 16s-R (Fig. 2; lanes 3 and 4). The 1555 A to $\mathrm{G}$ mutation deletes an $A l w 26$ I restriction enzyme cleavage site and so prevents cleavage by this enzyme (Fig. 2 ; lane 5). The Alw26I site is retained in the 519-bp amplification product from an individual who lacks the mutation, as shown by $A l w 26$ I cleavage to products of predicted size (Fig. 2; lane 6).

Using the multiplex AS-PCR for screening we found only $0.48 \%$ ( 1 of $206 ; 95 \%$ confidence interval, $0.01-2.75$ ) of a population of unrelated individuals from the province of Otago, New Zealand, possessed the mitochondrial $1555 \mathrm{~A}$ to $G$ mutation. This result suggests that the frequency of this mutation in New Zealand is similar to those of other populations studied.

This one-step multiplex AS-PCR approach is more rapid and economical for detection of single-base mutations than the combination of PCR amplification followed by restriction digestion. Multiplex AS-PCR is particularly helpful in detection of mutations such as the described mitochondrial A1555G mutation, which delete rather than create a restriction cleavage site. While the absence of cleavage provides an ambiguous result, the multiplex AS-PCR approach provides a definitive positive result as to the presence or absence of a specific mutation and is superior to duplex AS-PCR because it provides amplification in both the presence and absence of a given allele. We consider that the described approach will be of value in clinical assays where single-base mutations need to be detected.

\section{References}

El-Schahawi M, Lopez deMunain A, Sarrazin AM, Shanske AL, Basirico M, Shanske S, Dimauro S (1997) Two large Spanish pedigrees with nonsyndromic sensorineural deafness and the mtDNA mutation at nt 1555 in the 12s rRNA gene: evidence of heteroplasmy. Neurology 48:453-456

Estivill X, Govea N, Barcelo A, Perello E, Badenas C, Romero E, Moral L, Scozzari R, Durbano L, Zeviani M, Torroni A (1998) Familial progressive sensorineural deafness is mainly due to the mitochondrial DNA A1555G mutation and is enhanced by treatment with aminoglycosides. Am J Hum Genet 66:27-35

Fischel-Ghodsian N, Prezant TR, Chaltraw WE, Wendt KA, Nelson RA, Arnos KS, Falk RE (1997) Mitochondrial gene mutation is a significant predisposing factor in aminoglycoside ototoxicity. Am J Otolaryngol 18:173-178

Higashi K (1989) Unique inheritance of streptomycin induced deafness. Clin Genet 33:433-436

Hutchin T, Haworth I, Higashi K, Fischel-Ghodsian N, Stoneking M, Saha N, Arnos C, Cortopassi G (1993) A molecular basis for human hypersensitivity to aminoglycoside antibiotics. Nucleic Acids Res 21:4174-4179

Marthijs G, Claes S, Longo-Mbenza B, Cassiman JJ (1996) Nonsyndromic deafness associated with a mutation and a polymorphism in the mitochondrial 12s ribosomal RNA gene in a large Zairean pedigree. Eur J Hum Genet 4:46-51

Pandya A, Xia X, Radnaabazar J, Batsuuri J, Dangaansuren B, Fischel-Ghodsian N, Nance WE (1997) Mutation in the mitochondrial 12srRNA gene in two families from Mongolia with matrilineal aminoglycoside ototoxicity. J Hum Genet 34:169-172

Prezant TR, Agapian JV, Bohlman MC (1993) Mitochondrial ribosomal RNA mutation associated with both antibiotic-induced and nonsyndromic deafness. Nat Genet 4:289-294

Usami S, Abe S, Kasai M, Shinkawa H, Moeller B, Kenyon JB, Kimberling WJ (1997) Genetic and clinical features of sensorineural hearing loss associated with the 1555 mitochondrial mutation. Laryngoscope 107:483-490 